\title{
A bronchogenic cyst of the larynx: A case report
}

\author{
DAN LU*, RONG YU*, HUI YANG and JUN LIU \\ Department of Otorhinolaryngology, Head and Neck Surgery, West China Hospital, \\ Sichuan University, Chengdu, Sichuan 610041, P.R. China \\ Received January 3, 2017; Accepted July 20, 2017
}

DOI: 10.3892/etm.2017.5237

\begin{abstract}
A 12-year-old female presented with a 2-month history of dyspnea and a progressively worsening hoarseness lasting $>10$ years. The subsequently identified mass was completely resected from the patient's supraglottic area using a laser and suspension laryngoscope. The mass was diagnosed as a bronchogenic cyst using histopathology. While the prognosis for these cysts is good, long-term follow-up remains necessary for such patients because there are several reports in the literature indicating the potential for malignancy to develop. The patient in the present study experienced no complications or recurrence of the bronchogenic cyst 2 years following surgery. To the best of our knowledge, only 2 cases of bronchogenic cysts arising from the larynx have been reported in China, and the present study introduces the first case of a bronchogenic cyst of the larynx in a Chinese patient reported in English.
\end{abstract}

\section{Introduction}

Bronchogenic cysts are congenital lesions resulting from aberrant budding of the embryonic foregut (1). They may occur at any point along the tracheobronchial tree and are commonly localized in the mediastinum and lung parenchyma (2). It has previously been reported that bronchogenic cysts account for $10 \%$ of all mediastinal masses and are more common in males (3). However, bronchogenic cysts rarely occur in the larynx (4). To the best of our knowledge, only two cases of bronchogenic cysts arising from the larynx have been reported in China (4,5). The symptoms vary depending on the location and size of the cyst, typically including chronic cough, dyspnea, dysphagia, chest pain, hoarseness and increased stridor during sleeping (6). In the present study, a bronchogenic cyst that

Correspondence to: Professor Hui Yang, Department of Otorhinolaryngology, Head and Neck Surgery, West China Hospital, Sichuan University, 37 Guo Xue Xiang, Chengdu, Sichuan 610041, P.R. China

E-mail: yh8806@163.com

*Contributed equally

Key words: laryngeal cysts, larynx, bronchogenic cyst, congenital lesions, hoarseness developed in the supraglottic area of a 12-year-old female with consequent hoarseness and increased stidor is reported.

A 12-year-old female presented with a 2-month history of dyspnea and a progressively worsening hoarseness lasting $>10$ years. No other part of the patient's medical history was contributory. The patient was admitted to the Emergency Department of the West China Hospital, Sichuan University (Chengdu, China) with a grade 2 laryngeal obstruction (7) in May 2015. A flexible laryngoscopy revealed a large globular mass lesion with a smooth mucosal surface in the patient's supraglottic area, which obstructed around two-thirds of the patient's laryngeal cavity (Fig. 1). Physical examinations of the patient's neck and other otorhinolaryngological areas, analysis of the patient's blood biochemistry and radiographic analysis of the patient's chest were normal and unremarkable. A computed tomography (CT) scan of the patient's larynx revealed a large, well-defined cystic mass lesion measuring $3 \times 2 \times 1 \mathrm{~cm}$; as the lesion was located in the paralaryngeal space, between the patient's hyoid bone and thyroid gland, narrowing of the patient's glottic area was observed (Fig. 2). Since the large laryngeal lesion prevented intubation, a preventive tracheotomy was performed to secure an unobstructed airway, and then the lesion was excised using a laser and suspension laryngoscope (Fig. 3).

The vocal folds of the patient were protected by covering with wet saline gauze during the surgery and the cyst was determined to arise from the left ventricle of the larynx. The lesion was excised and identified to contain a clear yellow viscous liquid. Postsurgical histopathology revealed that the lesion was lined by a pseudostratified columnar epithelium and contained a section of the cyst wall, thus it was diagnosed as a bronchogenic cyst (Fig. 4). The staining procedure was performed as previously described. $(8,9)$. Tissue samples were fixed in $10 \%$ formalin for $10 \mathrm{~h}$ at room temperature and embedded in paraffin. Paraffin-embedded tissue specimens were cut to $3 \mu \mathrm{m}$ sections and stained with hematoxylin-eosin-saffron as previously described (9) and observed under a light microscope (magnification, x100). There were no complications during or following the surgery, and the patient was discharged from hospital 5 days after surgery. During the first follow-up 2 months after surgery, the patient's supraglottic and glottic structures were normal, and symmetrical movement of the vocal folds was observed. The patient's laryngeal cavity was demonstrated to be sufficiently large, thus the tracheal cannula was removed and the patient was able to breathe and speak normally (Fig. 5). The 2-year follow-up demonstrated that 


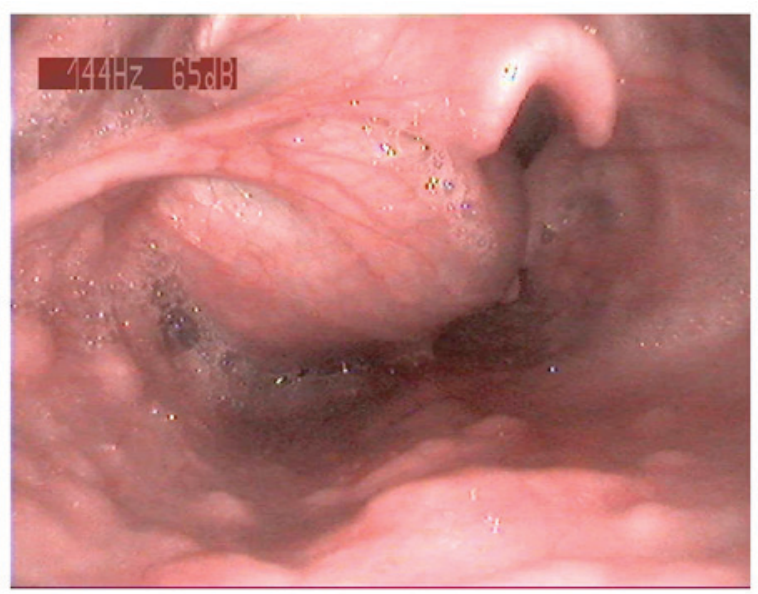

Figure 1. A flexible laryngoscopy revealing a large globular mass with a smooth mucosal surface in the supraglottic area.

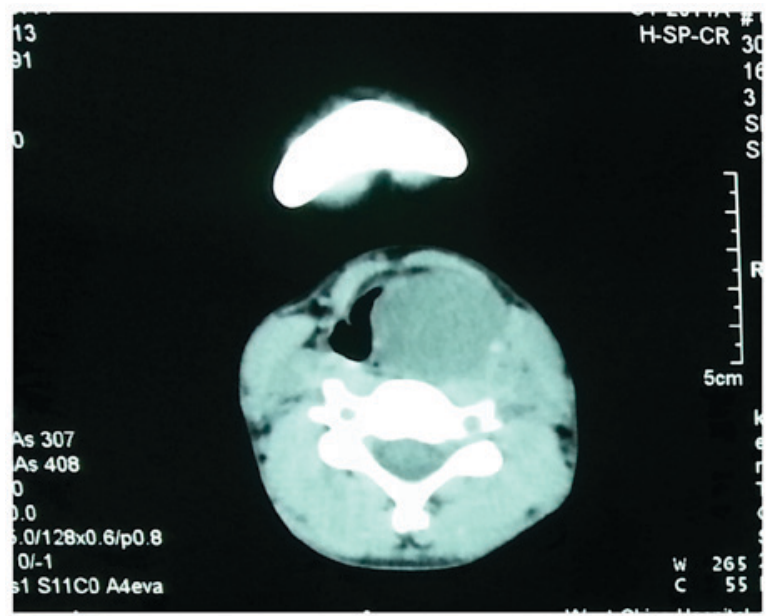

Figure 2. A presurgical computed tomography scan revealing a large, well-defined cystic lesion located in the paralaryngeal space.

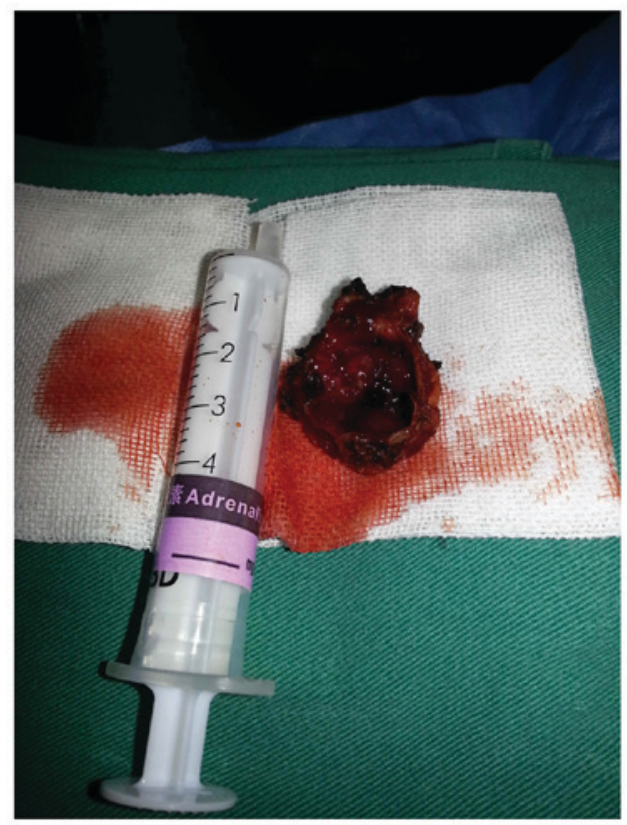

Figure 3. A mass measuring $3 \times 2 \times 1 \mathrm{~cm}$ was excised from the supraglottic area. The lesion was removed with a laser using a suspension laryngoscope.

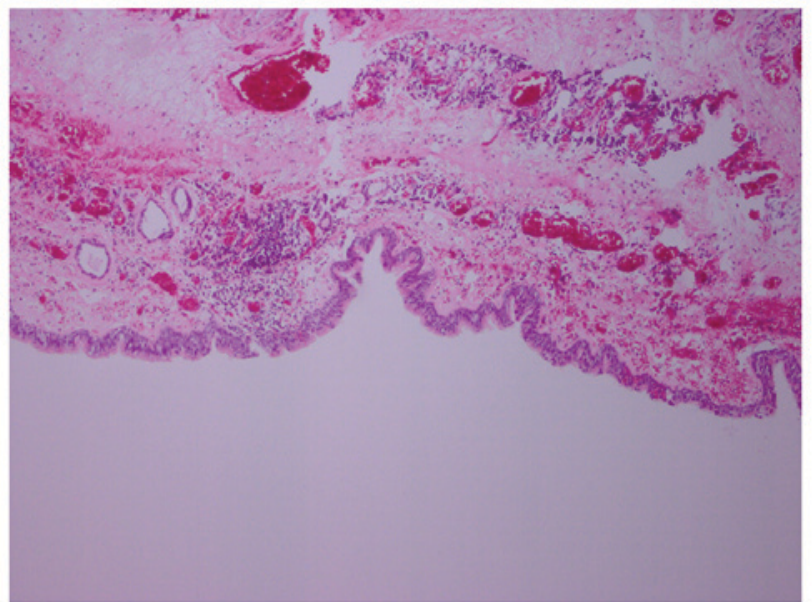

Figure 4. Histopathological analysis of the bronchogenic cyst. The section was stained with hematoxylin and eosin. Magnification, x100.

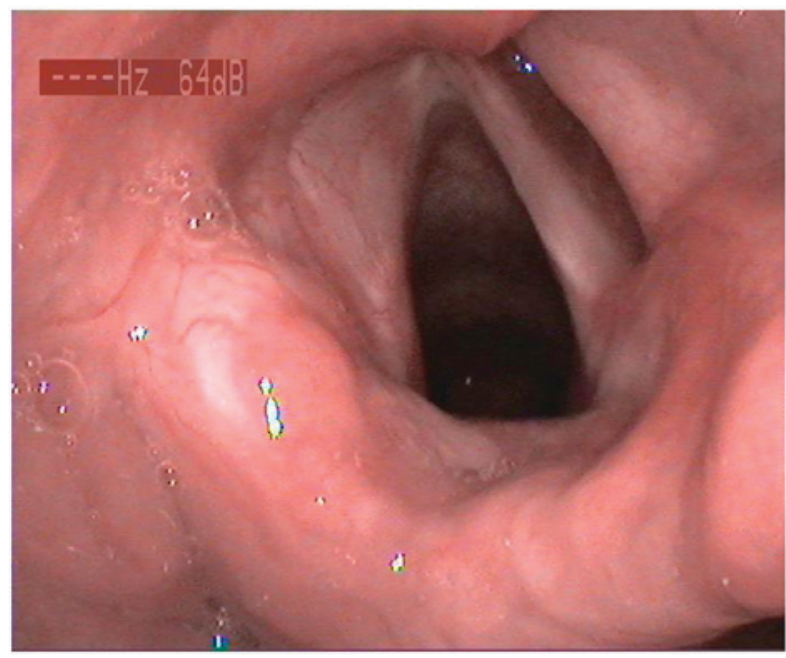

Figure 5. A postsurgical flexible laryngoscopy revealing a normal supraglottic and glottic structure 2 year after surgery. Prior to this image being captured the tracheal cannula was removed from the patient.

there was no recurrence of the bronchogenic cyst and that the patient exhibited normal vocal fold mobility. The patient provided written informed consent for their inclusion in the present study.

\section{Discussion}

Bronchogenic cysts are usually benign congenital lesions arising from abnormal budding of the ventral foregut, which forms between the third and sixth weeks of gestation. Although bronchogenic cysts are primarily located in the trachea and bronchi (10), they may also occur in other parts of the body, including the lungs, retroperitoneum, intrapericardial, anterior cranial fossa and neck (2,11-13). The first description of a laryngeal cyst was reported by Verneuil in 1852, and Abercrombie reported the next description in 1881 (14). Congenital laryngeal cysts are rare, occurring in 1.87/100,000 live births (15). Laryngeal cysts are the most common cysts located on the lingual surface of the epiglottis, followed by vallecular, ventricular and subglottic cysts (16). Bronchogenic 
cysts are congenital lesions that are rarely found in the larynx. Since patients with bronchogenic cysts do not typically present until the cyst is big enough to compress surrounding structures and thus cause symptoms, the true incidence of this type of disease is unknown. The clinical presentations of bronchogenic cysts vary depending on the location and size of the mass, and the age of the patient. In the present study, the patient had progressively worsening hoarseness and dyspnea because the increasing size of the cyst had caused compression of the vocal folds and glottis.

The accurate presurgical diagnosis of cysts can be difficult, and cysts may initially be misdiagnosed by radiologists and surgeons. The differential diagnosis for bronchogenic cysts includes congenital laryngeal cartilage dysplasia and pneumonia in infants, and sebaceous cysts, hemangiomas and solid tumors in adults. Ribet et al (17) reviewed the cases of 14 adults with bronchogenic cysts and identified that the presurgical misdiagnosis rate was $50 \%$. A combination of flexible laryngoscopy, CT, magnetic resonance imaging (MRI) and 2-(18F)-fluoro-2-deoxy-D-glucose positron emission tomography/CT can provide an assessment of the properties and location of the lesion, the degree of laryngeal constriction and the association of the lesion with surrounding vital structures (18). In CT images, bronchogenic cysts typically manifest as masses of soft tissue or water attenuation with distinct margins; MRI images can be useful in revealing the cystic nature of the mass. The majority of bronchogenic cysts have intermediate to higher signal intensity on T1-weighted images and characteristically exhibit high signal intensities on T2-weighted images (19). In the present study, CT images demonstrated a well-defined cystic lesion and as the cystic lesion was considered benign an MRI was not performed.

Histopathology is a technique used to aid in the diagnosis of bronchogenic cysts, which are typically cystic lesions lined by pseudostratified columnar epithelium, and often contain cartilage and bronchial mucus glands (20). The cysts are typically filled with clear fluid, hemorrhagic secretions or air, and occasionally calcium oxalate crystals are detected in the fluid (21). In the present study, the cyst contained a clear viscous liquid.

The treatment provided to patients with bronchogenic cysts is dependent on the location and size of the cyst, and the degree of respiratory comprise. In the past, a conservative 'watch-and-wait' treatment strategy was advocated for asymptomatic adults or high-risk patients (22); however, modern studies recommend surgical excision of the cyst to alleviate airway obstruction, and prevent possible infection (23), hemorrhage (24) and malignant degeneration (12). Therefore, once a bronchogenic cyst is suspected, surgical excision should be undertaken, even in an asymptomatic child (25). However, it is worth noting that sufficient preparation prior to surgery is very important for patients. If standard endotracheal intubation cannot be performed prior to surgery, a tracheotomy is required to alleviate airway obstruction. During surgery, the blood vessels, nerves and integrity of the laryngeal mucosa should be protected as far as possible, to ensure the quality of the patient's voice. Large obstructing cysts may be removed by the transoral route, but may require a lateral pharyngotomy or laryngofissure for excision.
Rather than transoral surgery, a minimally invasive surgery using a laser and suspension laryngoscope was performed in the present case to excise the lesion; this was performed for cosmetic reasons and to protect the vocal function of the patient. Following complete resection, the prognosis for bronchogenic cysts is good. However, long-term follow-up remains necessary for such patients because there are several reports in the literature indicating the malignant potential of bronchogenic cysts, including conversion into large cell carcinomas, bronchoalveolar carcinomas, adenocarcinomas, squamous cell carcinomas and metastasis to the lymph nodes (12). In the present case, the lesion was excised and the patient experienced no complications or recurrence of the bronchogenic cyst 2 year after surgery.

\section{Acknowledgements}

The present study was supported by the Sichuan Province Science and Technology Development Plan Item (grant nos. 2016FZ0106 and 2012FZ0014).

\section{References}

1. Borges AC, Knebel F, Lembcke A, Panda A, Komoda T, Hiemann NE, Meyer R, Baumann G and Hetzer R: Bronchogenic cyst of the interatrial septum presenting as atrioventricular block. Ann Thorac Surg 87: 1920-1923, 2009.

2. Castro R, Oliveira MI, Fernandes $T$ and Madureira AJ: Retroperitoneal bronchogenic cyst: MRI findings. Case Rep Radiol 2013: 853795, 2013.

3. Goswamy J, de Kruijf S, Humphrey G, Rothera MP and Bruce IA: Brochogenic cysts as a cause of infantile stridor: Case report and literature review. J Laryngol Otol 125: 1094-1097, 2011.

4. Chen YW, Gu DS and Wang TS: One case of bronchogenic cyst in larynx. Zhonghua Er Bi Yan Hou Tou Jing Wai Ke Za Zhi 46: 1045-1046, 2011 (In Chinese).

5. Zhou LJ, Zhang TS and Lin JY: A bronchogenic cyst in larynx: A case report. Chin Arch Otolaryngol Head Neck Surg 5: 307, 2003.

6. Williams HJ and Johnson KJ: Imaging of congenital cystic lung lesions. Paediatr Respir Rev 3: 120-127, 2002.

7. Wang LP, Zhang M, Li W, Tian Y, Xue XD and Wang SX: Etiologic analysis of severe neonatal upper respiratory tract obstruction. Zhonghua Er Bi Yan Hou Tou Jing Wai Ke Za Zhi 42: 753-756, 2007 (In Chinese).

8. Lepidi H, Casalta JP, Fournier PE, Habib G, Collard F and Raoult D: Quantitative histologic examination of mechanical heart valves. Clin Infect Dis 40: 655-661, 2005.

9. Lepidi H, Casalta JP, Fournier PE, Habib G, Collard F and Raoult D: Quantitative histologic examination of bioprosthetic heart valves. Clin Infect Dis 42: 590-596, 2006.

10. Shanmugam G, MacArthur K and Pollock JC: Congenital lung malformations-antenatal and postnatal evaluation and management. Eur J Cardiothorac Surg 27: 45-52, 2005.

11. Xu Q, Feng Y, Ye K, Zhou Y and Zhan R: Bronchogenic cyst in left anterior cranial fossa. Neurology 84: 1181-1182, 2015.

12. Jun HH, Kim SM, Lee YS, Hong SW, Chang HS and Park CS: Cervical bronchogenic cysts mimic metastatic lymph nodes during thyroid cancer surgery. Ann Surg Treat Res 86: 227-231, 2014.

13. Li Z, Wang X, Yang E, Gao K and Huang L: Gigantic intrapericardial bronchogenic cyst. Heart J 19: 532, 2011.

14. Lee WS, Tsai CS, Lin CH, Lee CC and Hsu HT: Airway obstruction caused by a congenital epiglottic cyst. Int J Pediatr Otorhinolaryngol 53: 229-233, 2000.

15. Pak MW, Woo JK and van Hasselt CA: Congenital laryngeal cysts: Current approacg to management. J Laryngol Otol 110: 854-856, 1996.

16. Saha D, Sinha R, Pai RR, Kumar A and Chakraborti S: Laryngeal cysts in infants and children - a pathologist's perspective (with review of literature). Int J Pediatr Otorhinolaryngol 77: 1112-1117, 2013. 
17. Ribet ME, Copin MC and Gosselin B: Bronchogenic cysts of the mediastinum. J Thorac Cardiovasc Surg 109: 1003-1110, 1995.

18. Yoon YR, Choi J, Lee SM, Kim YJ, Cho HD, Lee JW and Jeon YS: Retroperitoneal bronchogenic Cyst presenting paraadrenal tumor incidentally detected by (18)F-FDG PET/CT. Nuc Med Mol Imagi 49: 69-72, 2015.

19. Govaerts K, van Eyken P, Verswijvel G and Van der Speeten K: A bronchogenic cyst, presenting as a retroperitoneal cystic mass. Rare Tumors 4: e13, 2012.

20. Liang MK and Marks JL: Congenital bronchogenic cyst in the gastric mucosa. J Clin Pathol 58: 1344, 2005.

21. McAdams HP, Kirejczyk WM, Rosado-de-Christenson ML and Matsumoto S: Bronchogenic cyst: Imaging features with clinical and histopathologic correlation. Radiology 217: 441-446, 2000.
22. Tiwari MK, Yadav R, Mathur RM and Shrivastava CP: Mediastinal bronchogenic cyst presenting with dysphagia and back pain. Lung India 27: 86-88, 2010.

23. Hernández-Solís A, Cruz-Ortiz H, Gutiérrez-Díaz Ceballos ME and Cicero-Sabido R: Bronchogenic cysts. Importance of the infection in adults. Study of 12 cases. Cir Cir 83: 112-116, 2015 (In Spanis).

24. Kluger MD, Tayar C, Belli A, Salceda JA, van Nhieu JT, Luciani A and Cherqui D: A foregut cystic neoplasm with diagnostic and therapeutic similarities to mucinous cystic neoplasms of the pancreas. JOP 14: 446-449, 2013.

25. Stewart B, Cochran A, Iglesia K, Speights VO and Ruff T: Unusual case of stridor and wheeze in an infant: Tracheal bronchogenic cyst. Pediatr Pulmonol 34: 320-323, 2002. 